\title{
Eleven Surveys in Operations Research: II
}

\author{
Denis Bouyssou · Silvano Martello · Frank Plastria
}

Received: 23 April 2009

\section{Foreword}

This volume of Annals of Operations Research collects all eleven survey papers that appeared in volumes 4-6 (2006-2008) of 4OR. This is the second volume of this type after Bouyssou et al. (2007a).

The wide range of topics covered, the depth of each coverage, the timeliness of each of the topics, and the prestigious authors who contributed to them, have prompted the editors of this upcoming journal to make them more widely available through this channel.

The authors of these survey papers were given the opportunity to revise their texts, updating them to include the most recent relevant material. The editors hereby very warmly thank them all for their initial contribution and their willingness to update it on short notice.

\section{About $40 R$}

$4 O R$ is a quarterly journal jointly published by the Belgian (SOGESCI/ BVWB), French (ROADEF) and Italian (AIRO) Operations Research societies. All members of SOGESCI/ BVWB, ROADEF and AIRO receive the journal as part of their membership package. As its name suggests, this journal is 'for' Operations Research (OR).

$4 O R$ was started in 2002 under the subtitle 'Quarterly Journal of the Belgian, French and Italian Operations Research Societies'. The growing international impact of the journal

\section{Denis Bouyssou}

CNRS-LAMSADE, UMR 7024, Université Paris Dauphine, Place du Maréchal de Lattre de Tassigny, F-75775 Paris Cedex 16, France, tel: +33 1440548 98, fax: +33 1440540 91, E-mail: 4or@lamsade.dauphine.fr

Silvano Martello

DEIS, Università di Bologna, Viale Risorgimento 2, 40136 Bologna, Italy tel: +39 051 2093022, fax: +39 051 2093073, E-mail: dipdeis.4or@unibo.it

Frank Plastria

MOSI, Department of Mathematics, Operational Research, Statistics and Information Systems for Management, Vrije Universiteit Brussel, Pleinlaan 2, B-1050 Brussels, Belgium, tel: +32 2 6293609, fax: +32 2 6293690, E-mail: 4or.be@vub.ac.be 
was reflected by a change in its name: starting with Issue 2 of Volume 3, it is now " $4 O R$, A Quarterly Journal of Operations Research".

$4 O R$ replaced JORBEL, the former journal of the Belgian OR society, and Ricerca $O$ perativa, the former journal of the Italian OR society, while the French OR society did not publish any journal before the creation of $4 O R$. Both JORBEL and Ricerca Operativa demanded a great deal of effort from their editors, while having a distribution mainly limited to Belgium and Italy respectively. The creation of $4 O R$ answered the need for a much more efficient use of scarce resources. An account of the objectives of the journal can be found in Bouyssou et al. (2003). A report on the first three years of existence of the journal is given in Bouyssou et al. (2006a). A similar report for the period 2006-2008 is presented in Bouyssou et al. (2009a).

$4 O R$ is managed by a group of three editors-in-chief, one from each promoting OR society, assisted by a board of area editors. The three authors of this text have acted as editors-in-chief of $4 O R$ since its creation. A new board of editors-in-chief was appointed at the end of 2008, Philippe Baptiste replacing Denis Bouyssou. This change was accompanied by the appointment of a new board of area editors.

$4 O R$ has been accepted in ISI's Science Citation Index Expanded (SCIE). The journal will be covered in the 2008 ISI report (this report will be published in June 2009). 4OR will receive an impact factor from 2009. The first impact factor of our journal will be published in 2010 .

$4 O R$ has an on-line edition (http://wwww . springerlink . com/content/111812/) that is included in most journal bundles offered by Springer to libraries. This is a major asset for the journal since being clearly visible on the web is a necessary condition for a journal to have a reasonable chance of surviving. Furthermore, accepted papers are available for download on the site, under Online First ${ }^{\mathrm{TM}}$, (and have a DOI) as soon as the proofs are corrected. This creates a very nice compromise between the wishes of the editors of the journal to have a reasonable backlog of papers and those of authors willing to see their papers published as soon as possible.

Every year one volume of four issues, each of around 100 pages, is published containing papers in five different sections with different frequencies (between brackets the number of each of them over volumes 4-6):

Invited surveys (11): state-of-the-art surveys written by prominent researchers on invitation by the editors, limited to one paper per issue.

Research papers (27): original research papers, with a preference for relatively short ones (around 12-15 pages, the limits being fuzzy). The journal publishes papers in all areas of OR. Papers in emerging fields of OR are especially welcomed.

$\mathrm{PhD}$ thesis abstracts (28): 3 or 4-page summaries of $\mathrm{PhD}$ Theses defended in Belgium, France and Italy, or by Belgian, French and Italian students studying abroad, published under the responsibility of their advisors.

Industry (6): case studies, state-of-the-art papers on the applications of OR techniques or reflections on the practice of $\mathrm{OR}$.

Educational papers (0): up-to-date expositions of classical OR problems that are frequently used in OR classes, or papers throwing a novel light on such.

All papers are exchanged and processed in electronic form. The refereeing process conforms with the usual standards of academic journals. After having passed a first quick scan for appropriateness and level, it involves at least two independent referee reports and the advice of an appropriate area editor. Except for two cases of plagiarism that were fortunately detected (see Bouyssou et al. 2006b 2009b), the reviewing process of the papers was rather smooth. 
The collaboration between the three editors and the area editors proved effective and efficient. Our purely electronic way of handling papers turned out to be swift and economical.

The journal has an uncommon policy with respect to major revisions. Papers needing a minor revision will not normally be sent back in revised form to the referees and the (area) editors will check their adequacy to the suggestions of the referees. On the contrary, all papers needing a 'major revision', i.e., a revision that cannot be reasonably expected to be undertaken within two months and/or that is likely to significantly alter the whole paper, will be rejected. Needless to say, papers rejected because they need a major revision can-after substantial rewriting - be resubmitted to $4 O R$. They are then considered as new submissions and, thus, evaluated by two, possibly new, referees.

For volumes 4-6, the rejection rate for research papers was $79 \%$. The mean time between the reception of the paper and the communication of the decision to the authors was 144 days, i.e., less than 5 months. We received submissions coming from 28 different countries in the world.

\section{The surveys in this volume}

Invited surveys are solicited by one of us and are reviewed collectively by the three of us. We have tried hard to solicit papers dealing with important or emerging fields of OR. Furthermore, we have strived to obtain papers from authors coming from a large variety of countries. Our policy is to solicit surveys written by well-established scholars, presenting the state-of-the-art of relevant Operations Research areas.

In this section we briefly introduce each of the surveys collected in this volume. The papers have been kept in their original order of publication, this being the only rational and fair order for such a wide range of subjects. This order may be observed from the $4 O R$ volume/issue number (shown in brackets) in which they originally appeared.

Some operations research methods for analyzing protein sequences and structures (4OR 4/2, Błażewicz et al. (2006)): Jacek Błażewicz, Piotr Łukasiak and Maciej Miłostan review Operations Research techniques that were relatively recently introduced in biology, especially in the protein analysis area, to support biologists.

Integrating operations research in constraint programming (4OR 4/3, Milano and Wallace (2006)): Michela Milano and Mark Wallace describe how constraint programming, viewed as a natural formalism for modeling problems, allows linear programming to be combined with propagation and novel and varied search techniques.

Ejection chain and filter-and-fan methods in combinatorial optimization $(4 O R$ 4/4, Glover and Rego (2006)): Fred Glover and César Rego present the general framework of the ejection chain and filter-and-fan methods, powerful neighborhood structures fundamental to the performance of local search and metaheuristic algorithms for combinatorial optimization.

An updated survey on the linear ordering problem for weighted or unweighted tournaments (4OR 5/1, Charon and Hudry (2007)): Irène Charon and Olivier Hudry survey results, conjectures and open problems dealing with the combinatorial and algorithmic aspects of the linear ordering problem.

Combinatorial optimization and green logistics (4OR 5/2, Sbihi and Eglese (2007)): Abdelkader Sbihi and Richard W. Eglese introduce the area of green logistics and describe the main topics in this field which can be formulated as combinatorial optimization problems.

Generalized Nash equilibrium problems (4OR 5/3, Facchinei and Kanzow (2007)): Francisco Facchinei and Christian Kanzow discuss the main properties and solution algorithms 
for the generalized Nash equilibrium problem, an important model that has its roots in the economic sciences but is being fruitfully used in many different fields.

Exact algorithms for routing problems under vehicle capacity constraints (4OR 5/4, Baldacci et al. (2007)): Roberto Baldacci, Paolo Toth and Daniele Vigo review the most recent developments that had a major impact in the current state-of-the-art of exact algorithms for the capacitated vehicle routing problem.

A decade of application of the Choquet and Sugeno integrals in multi-criteria decision aid (4OR 6/1, Grabisch and Labreuche (2008)): Michel Grabisch and Christophe Labreuche examine the main advances on the use of the Choquet and Sugeno integrals in multi-criteria decision aid over the last decade.

On the use of graphs in discrete tomography (4OR 6/2, de Werra et al. (2008)): Dominique de Werra, Marie-Christine Costa, Christophe Picouleau and Bernard Ries present a tutorial paper on a graph theoretical model for the basic image reconstruction problem which stems from discrete tomography.

Mathematical optimization in intensity modulated radiation therapy (4OR 6/3, Ehrgott et al. (2008)): Matthias Ehrgott, Çiğgdem Güler, Horst W. Hamacher and Lizhen Shao survey optimization models, methods, and theories in mathematical optimization which were recently successfully introduced in the design of intensity modulated radiotherapy treatments.

Variable neighborhood search: methods and applications (4OR 6/4, Hansen et al. (2008)): Pierre Hansen, Nenad Mladenović and José Moreno Pérez review the main aspects of variable neighborhood search metaheuristics, a framework based upon systematic changes of neighborhoods.

\section{The first AOR volume}

We take this occasion to briefly present the surveys originally published in $4 O R$ that were included in the first Annals of Operations Research volume (Bouyssou et al.2007a) referring the reader to Bouyssou et al. (2007b) for a more detailed description.

The omnipresence of Lagrange (4OR 1/1, Lemaréchal (2003)): Claude Lemaréchal points out that Lagrangean relaxation is not just a technique for handling combinatorial optimization problems, but a basic method in many more optimization issues.

The dial-a-ride problem: Variants, modeling issues and algorithms (4OR 1/2, Cordeau and Laporte (2003)): Jean-François Cordeau and Gilbert Laporte present an exhaustive introduction to door-to-door delivery problems arising in social services.

Lifting, superadditivity, mixed integer rounding and single node flow sets revisited (4OR 1/3, Louveaux and Wolsey (2003)): Quentin Louveaux and Laurence Wolsey show that a combination of integer rounding and lifting techniques provides a unified way to obtain many of the valid inequalities for mixed 0-1 sets that have been proposed in the literature.

Models and solution techniques for frequency assignment problems (4OR 1/4, Aardal et al. (2003)): Karen Aardal, Stan van Hoesel, Arie Koster, Carlo Mannino and Antonio Sassano give a thorough overview of the different modeling ideas developed for the various situations in which a frequency assignment problem arises in wireless communication.

Combinatorial auctions (4OR 2/1, Abrache et al. (2004)): Jawad Abrache, Teodor Gabriel Crainic, Michel Gendreau and Monia Rekik review several contributions from the Operations Research literature on a particular type of auctions in which participants are allowed to bid on bundles of multiple heterogeneous items.

Ethics in OR/MS: Past, present and future (4OR 2/2, Brans and Gallo (2004)): JeanPierre Brans and Giorgio Gallo discuss fundamental questions on the social role of Opera- 
tions Research / Management Science tools and the new challenges posed by the growing complexity of the problems which humanity is facing today.

Combinatorial optimization and hierarchical classifications (4OR 2/3, Barthélemy et al. (2004)): Jean-Pierre Barthélemy, François Brucker and Christophe Osswald review several extensions of traditional hierarchical classification techniques from a combinatorial optimization point of view, and study the complexity of the underlying optimization problems.

Counting and enumeration complexity with application to multicriteria scheduling (4OR 3/1, T'kindt et al. (2005)): Vincent T'kindt, Karima Bouibede-Hocine and Carl Esswein study several aspects of multicriteria scheduling, with special emphasis on the complexity of problems linked with counting and enumerating optimal solutions.

Bilevel programming: A survey (4OR 3/2, Colson et al. (2005)): Benoît Colson, Patrice Marcotte and Gilles Savard review the good progress that is being made in handling special classes of problems in which the objective involves the optimal solution to another second level problem, or admit even more levels.

Complexity and algorithms for convex network optimization and other nonlinear problems (4OR 3/3, Hochbaum (2005)): Dorit Hochbaum analyzes the effort needed to produce optimal solutions with pre-specified accuracy for nonlinear continuous optimization problems, and presents polynomial-time methods for several nonlinear network problems.

Production planning with load dependent lead times (4OR 3/4, Pahl et al. (2005)): Julia Pahl, Stefan Voß and David L. Woodruff give a detailed account of techniques for supply chain management in contexts where it is required to produce goods in a timely manner so as to be able to meet a demand varying in time and space.

We believe that the 22 surveys published in the first 6 years of existence of $4 O R$ offer an in-depth coverage of many "hot fields" in Operations Research. We clearly intend to continue to publish such thorough invited surveys in future issues of $4 O R$.

\section{References}

K. I. Aardal, S. P. M. van Hoesel, A. M. C. A. Koster, C. Mannino, and A. Sassano. Models and solution techniques for frequency assignment problems. 4OR, 1(4):261-317, 2003.

J. Abrache, T. G. Crainic, and M. Gendreau. Design issues for combinatorial auctions. 4OR, 2(1):1-33, 2004.

R. Baldacci, P. Toth, and D. Vigo. Recent advances in vehicle routing exact algorithms. 4OR, 5(4):269-298, 2007.

J.-P Barthélemy, F. Brucker, and C. Osswald. Combinatorial optimization and hierarchical classifications. 4OR, 2(3):179-219, 2004.

J. Błażewicz, P. Łukasiak, and M. Miłostan. Some operations research methods for analyzing protein sequences and structures. 4OR, 4(2):91-123, 2006.

D. Bouyssou, S. Martello, and F. Plastria. 4OR for what purpose? 4OR, 1(1):1-6, 2003.

D. Bouyssou, S. Martello, and F. Plastria. 4OR, Year 4. 4OR, 4(1):1-9, $2006 \mathrm{a}$.

D. Bouyssou, S. Martello, and F. Plastria. A case of plagiarism: Dănuţ Marcu. 4OR, 4(1):11-13, $2006 \mathrm{~b}$.

D. Bouyssou, S. Martello, and F. Plastria. (eds.) Surveys in Operations Research (Invited surveys from 4OR), volume 153 of Annals of Operations Research. Springer, New York, 2007a.

D. Bouyssou, S. Martello, and F. Plastria. Eleven surveys in operations research. Annals of Operations Research, 153(1):3-7, 2007b.

D. Bouyssou, S. Martello, and F. Plastria. No 7-year itch at 4OR. 4OR, 7(1):1-15, 2009a.

D. Bouyssou, S. Martello, and F. Plastria. Plagiarism again: Sreenivas and Srinivas, with an update on Marcu. 4OR, 7(1):17-20, 2009b.

J.-P. Brans and G. Gallo. Ethics in OR/MS: Past, present and future. 4OR, 2(2):95-110, 2004.

I. Charon and O. Hudry. A survey on the linear ordering problem for weighted or unweighted tournaments. 4OR, 5(1):5-60, 2007.

B. Colson, P. Marcotte, and G. Savard. Bilevel programming: A survey. 4OR, 3(2):87-107, 2005.

J.-F. Cordeau and G. Laporte. The dial-a-ride problem: Variants, modeling issues and algorithms. 4OR, 1(2): 89-101, 2003. 
D. de Werra, M.-C. Costa, C. Picouleau, and B. Ries. On the use of graphs in discrete tomography. $4 O R, 6$ (2):101-123, 2008.

M. Ehrgott, Ç. Güler, H.W. Hamacher, and L. Shao. Mathematical optimization in intensity modulated radiation therapy. 4OR, 6(3):199-262, 2008.

F. Facchinei and C. Kanzow. Generalized Nash equilibrium problems. 4OR, 5(3):173-210, 2007.

F. Glover and C. Rego. Ejection chain and filter-and-fan methods in combinatorial optimization. 4OR, 4(4): 263-296, 2006.

M. Grabisch and C. Labreuche. A decade of application of the Choquet and Sugeno integrals in multi-criteria decision aid. 4OR, 6(1):1-44, 2008.

P. Hansen, N. Mladenović, and J. Moreno Pérez. Variable neighborhood search: Methods and applications. 4OR, 6(4):99-999, 2008.

D. S. Hochbaum. Complexity and algorithms for convex network optimization and other nonlinear problems. 4OR, 3(3):171-216, 2005

C. Lemaréchal. The omnipresence of Lagrange. 4OR, 1(1):7-25, 2003.

Q. Louveaux and L. A. Wolsey. Lifting, superadditivity, mixed integer rounding and single node flow sets revisited. 4OR, 1(3):173-207, 2003.

M. Milano and M. Wallace. Integrating operations research in constraint programming. 4OR, 4(3):175-219, 2006.

J. Pahl, S. Voß, and D. L. Woodruff. Production planning with load dependent lead times. 4OR, 3(4):257-302, 2005

A. Sbihi and R.W. Eglese. Combinatorial optimization and green logistics. 4OR, 5(2):99-116, 2007.

V. T'kindt, K. Bouibede-Hocine, and C. Esswein. Counting and enumeration complexity with application to multicriteria scheduling. 4OR, 3(1):1-21, 2005. 